\title{
Compared with parenteral nutrition, enteral feeding attenuates the acute phase response and improves disease severity in acute pancreatitis
}

\author{
A C J Windsor, S Kanwar, A G K Li, E Barnes, J A Guthrie, J I Spark, F Welsh,
} P J Guillou, J V Reynolds

\begin{abstract}
Background-In patients with major trauma and burns, total enteral nutrition (TEN) significantly decreases the acute phase response and incidence of septic complications when compared with total parenteral nutrition (TPN). Poor outcome in acute pancreatitis is associated with a high incidence of systemic inflammatory response syndrome (SIRS) and sepsis.

Aims-To determine whether TEN can attenuate the acute phase response and improve clinical disease severity in patients with acute pancreatitis.
\end{abstract}

Methods-Glasgow score, Apache II, computed tomography (CT) scan score, $C$ reactive protein (CRP), serum IgM antiendotoxin antibodies (EndoCAb), and total antioxidant capacity (TAC) were determined on admission in 34 patients with acute pancreatitis. Patients were stratified according to disease severity and randomised to receive either TPN or TEN for seven days and then re-evaluated. Results-SIRS, sepsis, organ failure, and ITU stay, were globally improved in the enterally fed patients. The acute phase response and disease severity scores were significantly improved following enteral nutrition (CRP: $156(117-222)$ to 84 (50141), p<0.005; APACHE II scores 8 (6-10) to $6(4-8), p<0.0001)$ without change in the CT scan scores. In parenterally fed patients these parameters did not change but there was an increase in EndoCAb antibody levels and a fall in TAC. Enterally fed patients showed no change in the level of EndoCAb antibodies and an increase in TAC.

Conclusion-TEN moderates the acute phase response, and improves disease severity and clinical outcome despite unchanged pancreatic injury on CT scan. Reduced systemic exposure to endotoxin and reduced oxidant stress also occurred in the TEN group. Enteral feeding modulates the inflammatory and sepsis response in acute pancreatitis and is clinically beneficial.

(Gut 1998;42:431-435)

Keywords: acute pancreatitis; enteral nutrition; bacterial translocation; oxidative stress

Most morbidity and deaths from acute pancreatitis are consequences of the immunoin- flammatory response to pancreatic necrosis or infection. ${ }^{12}$ This response manifests as a spectrum of disease, with clinical progression from the systemic inflammatory response syndrome (SIRS), to sepsis, multiple organ failure (MOF), and death. ${ }^{3}$ The gastrointestinal tract is increasingly seen as a potential source of fuel for such an immunoinflammatory response in critically ill patients because of experimental data suggesting that the endogenous cytokines responsible for this response are stimulated by endotoxin and other bacterial products absorbed by a metabolically deprived gut. ${ }^{4}$ This concept of mucosal injury has also been invoked as a cause of the inflammatory response in experimental acute pancreatitis. ${ }^{56}$ In parallel with this concept, several studies have shown a reduction in septic morbidity following early enteral feeding in other immunoinflammatory conditions associated with trauma, ${ }^{7}$ thermal injury, ${ }^{8}$ and major surgery. ${ }^{9} \mathrm{~A}$ proposed mechanism for these improved clinical outcomes suggests that feeding the gut maintains normal gut barrier function against the translocation of luminal bacteria and toxins. Moreover, in contrast to parenteral nutrition, enteral feeding seems to modulate the acute phase response and preserves visceral protein metabolism, suggesting downregulation of the splanchnic cytokine response..$^{10}$

Conventional management of acute pancreatitis is based on gut rest, with or without the provision of total parenteral nutrition. ${ }^{12}{ }^{13}$ However, given the experimental data on the role of the gut in acute pancreatitis, together with the suggestion that enteral nutrition may diminish septic complications in critically ill patients, there seems to be some logic in considering use of the enteral rather than the parenteral route for nutritional support in patients with acute pancreatitis. ${ }^{14} \mathrm{~A}$ recent report has suggested that enteral nutrition is safe and may promote more rapid resolution of toxicity in patients with mild acute pancreatitis. ${ }^{15}$ Furthermore a preliminary report on 11 patients with acute pancreatitis who required surgery and had a feeding jejunostomy implanted, failed to reveal any detrimental effect on the clinical or radiological course of acute pancreatitis when the patients were fed by this route. ${ }^{16}$ Given the potential benefits of enteral nutritional support within other scenarios, the purpose of this study was to determine the feasibility of enteral nutrition in patients suffering from acute pancreatitis and to examine biological parameters relating to 


\begin{tabular}{|c|c|c|c|}
\hline Enrolment & Nutritional support & Discharge & Follow up \\
\hline$\checkmark$ & $\checkmark$ & $\checkmark$ & $\checkmark$ \\
\hline $48 \mathrm{~h}$ & 7 days & & \\
\hline$\uparrow$ & $\begin{array}{l}\text { Acute physiology } \\
\text {-Glasgow score } \\
\text {-APACHE II }\end{array}$ & & \\
\hline & $\begin{array}{l}\text { Serum } \\
\text {-C reactive protein } \\
\text {-EndoCAb antibodies } \\
\text {-Total antioxidant capacity }\end{array}$ & \multirow{2}{*}{\multicolumn{2}{|c|}{$\begin{array}{l}\text { Clinical endpoints } \\
\text {-Hospital stay } \\
\text {-SIRS, sepsis, MOF } \\
\text {-Intervention } \\
\text {-Mortality }\end{array}$}} \\
\hline & $\begin{array}{l}\text { Radiology } \\
\quad-\text { CT score (modified Balthazar) }\end{array}$ & & \\
\hline
\end{tabular}

the theoretical mode of action of enteral nutrition within the context of a randomised clinical trial of enteral versus parenteral nutrition in acute pancreatitis.

\section{Patients and methods}

Between February 1995 and March 1996 a randomised clinical study of enteral versus parenteral nutrition in acute pancreatitis was undertaken. Patients with a serum amylase of greater than $1000 \mathrm{IU}$ and clinical evidence of acute pancreatitis were enrolled. Patients without clinical evidence of acute pancreatitis, patients with known chronic pancreatitis, and those presenting more than 48 hours after admission were excluded from the study. Patients were then stratified according to their admission Glasgow score. ${ }^{17}$ Three or more Glasgow points indicates severe disease and less than three mild/moderate disease. All patients were then randomised according to odd or even hospital number to receive either enteral or parenteral feeding. In the severe group, parenteral feeding was delivered through a central venous catheter (Hydrocath Triple lumen, Ohmeda, Swindon), and enteral feeding through a radiologically placed nasojejunal feeding tube (Corsafe tube, Corpak Medsystems, Merck Ltd, Hants). In the mild/moderate group patients were similarly randomised to receive parenteral feeding through a peripheral long line (Hydrocath Mid-line, Ohmeda, Swindon) placed in the antecubital fossa, or enteral feeding in the form of oral nutritional supplements. All intravenous catheters were sent for microbiological examination on removal.

The standard parenteral formula used in both mild/moderate and severe groups was Kabi regimen 1 (Pharmacia and Upjohn, Knowhill, Milton Keynes); $2500 \mathrm{ml}$ provides $9.4 \mathrm{~g}$ nitrogen and 7.52 non-protein $\mathrm{MJ}$ (8.57 total MJ) per 24 hours. Lipid contributed $55 \%$ of the non-protein calories. This solution was formulated on site in the pharmacy and contained vitamins and trace elements. All peripherally delivered parenteral solutions also contained 1500 IU heparin.

In the severe group the standard enteral feed used was Osmolite (Ross Products, Queensborough, Kent) providing $12 \mathrm{~g}$ nitrogen and 7.11 non-protein $\mathrm{MJ}$ (8.44 total MJ) in 2000 $\mathrm{ml}$ per 24 hours once established. Of the nonprotein energy $36 \%$ was lipid based. The enteral feed was introduced at $30 \mathrm{ml} / \mathrm{h}$ and the rate increased incrementally, depending on tolerance, at up to $100 \mathrm{ml} / \mathrm{h}$. The regimen was based on four, five hourly feeds with one hour rest between each.

The oral diet given to patients with mild/ moderate acute pancreatitis consisted of clear fluids with nutritional supplements, such as Entera (Fresenius, Runcorn, Cheshire) providing $1.56 \mathrm{~g}$ nitrogen and 1.07 non-protein $\mathrm{MJ}$ (1.25 total $\mathrm{MJ}$ ) per carton and Fortisip (Nutricia Clinical Care, Trowbridge, Wilts) providing $1.8 \mathrm{~g}$ nitrogen and 1.09 non-protein $\mathrm{MJ}(1.25$ total $\mathrm{MJ}$ ) per carton. Up to five cartons of supplement were taken each day, but no attempt was made to enforce an isoenergic, isonitrogenous intake in the enterally fed compared with the parenterally fed groups. Fluid requirements over and above those delivered in the form of nutritional support were provided intravenously in the form of crystalloid and colloid solutions or clear fluids depending on clinical criteria.

The study protocol was divided into four study periods (fig 1). An initial 48 hour enrolment period was followed by a seven day nutritional support period. The third time period was the time from the nutritional support period to discharge and the fourth from discharge to clinical follow up. Within the first 48 hour enrolment period patients were evaluated for aetiology and Glasgow and APACHE II scores ${ }^{18}$ and serum $\mathrm{C}$ reactive protein (CRP) was measured. Serum was also stored at $-70^{\circ} \mathrm{C}$ for subsequent measurement of $\operatorname{IgM}$ anticore endotoxin antibodies (EndoCAb, Chromogenix, Molndal, Sweden) using an enzyme linked immunosorbent assay (ELISA) technique. ${ }^{19}$ Total antioxidant potential (TAC) was quantified using an enhancement chemiluminescence assay. ${ }^{20}$ Patients then underwent a dynamic contrast enhanced comupted tomography (CT) scan. These scans were scored by a consultant radiologist using a modified Balthazar scoring system. ${ }^{21} 22$ This score defines pancreatic and peripancreatic morphology in addition to degree of glandular necrosis, defined by enhancement values of below 30 Hounsfield units. The radiologist was blinded to the nature of nutritional support being administered. Patients then received their seven day nutritional support according to randomisation. On completion of their nutritional support period, patients were re-evaluated in an identical fashion to that outlined above for the clinical, radiological, and laboratory parameters. Following this, continuation of nutritional support or reintroduction of a normal dietary intake was undertaken according to the wishes of the attending clinician.

Patients were monitored during the study until hospital discharge and then during regular clinical follow up for outcome endpoints including: SIRS and intra-abdominal sepsis, defined by Bone's criteria ${ }^{23}$; the incidence of multiple organ failure as defined by Tran et $\mathrm{al}^{24}$; the need for operative intervention; hospital and intensive care unit stay; and 30 day 
Table 1 Patient demographics and disease severity at enrolment

\begin{tabular}{lccl}
\hline & Enteral & Parenteral \\
\hline Patient numbers & 16 & 18 & \\
Age (y) & $63(47-76)$ & 63 & $(52-73)$ \\
Sex & $9(\mathrm{~F}) 7(\mathrm{M})$ & 11 & $(\mathrm{~F}) 7(\mathrm{M})$ \\
Disease severity & 6 & 7 & \\
$\quad$ Severe & 6 & 11 & \\
$\quad$ Mild/moderate & $2(1-3)$ & 2 & $(1-4)$ \\
Glasgow score & $156(117-222)$ & $125(49-168)$ \\
CT score & $3(2.5-5)$ & $4.5(2.5-5.5)$ \\
C reactive protein & $8(6-10)$ & $9.5(8-13)$ \\
APACHE II score & $38(38-41) \mathrm{g} / 1$ & $35.5(30-36.5) \mathrm{g} / 1$ \\
Serum albumin on admission & $29(28-37) \mathrm{g} / 1$ & 30 & $(26.5-35) \mathrm{g} / 1$ \\
Serum albumin at 7 days & & & \\
Causes of pancreatitis & 9 & 14 & \\
$\quad$ Gallstones & 2 & 2 & \\
Alcohol & 3 & 1 & \\
ERCP & 1 & 1 &
\end{tabular}

ERCP, endoscopic retrograde cholangiopancreatography.

mortality. Antibiotics were not routinely prescribed but were introduced on a secondary rise in CRP or microbiologically proven infection. The indications for operation included persistent or deteriorating organ failure despite maximal intensive care for at least three days; verified infected pancreatic necrosis; and large, symptomatic, or infected pseudocyst formation.

The primary endpoint of the study was the incidence of the systemic inflammatory response syndrome, with sepsis, organ failure, hospital stay, and mortality as secondary endpoints. Data are expressed as medians with interquartile ranges. Statistical analysis between groups was performed using the MannWhitney $U$ test for non-categorical data and the $\chi^{2}$ test with Yates's correction for categorical data. Within group analysis was undertaken with Wilcoxon signed rank, matched pairs analysis. Statistical significance was accepted at a $p$ value of less than 0.05 .

\section{Results}

Thirty four consecutive patients with pancreatitis fulfilled the enrolment criteria. Sixteen were randomised to enteral feeding and 18 to parenteral feeding. Both groups were matched for age, sex, and severity stratification (table 1).
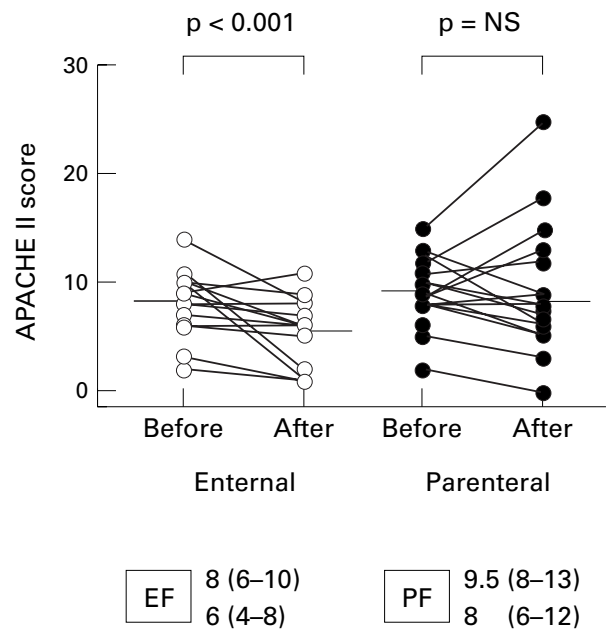

Comparison of disease severity between the groups on entry revealed no significant differences in terms of APACHE II score, Glasgow score, CT score, and serum CRP (table 1). No patient randomised to the enteral feeding group required conversion to parenteral nutrition. However, in five patients in the enterally fed group who developed an ileus, temporary reduction in the volume of their enteral nutrition was required for between two and four days because of nausea and fullness. In both patients resumption of full enteral support was acheived for the remainder of the study period. In addition one tube blocked and another was accidentally displaced but both were immediately repositioned and feeding resumed. No patient in the enterally fed group experienced diarrhoea.

The median amount of non-protein energy delivered in the enterally fed group per patient per day was 5.02 MJ (range 3.25-6.02) whereas in the parenterally fed group all 7.52 non-protein $\mathrm{MJ}$ were delivered daily to all patients. This difference was statistically significant $(p<0.0004)$. The corresponding nitrogen delivery in the two groups was 9.24 (5.9511.0) g per patient per day in the enterally fed group and $9.4 \mathrm{~g}$ per patient per day in the parenterally fed group ( $\mathrm{p}=0.08, \mathrm{NS})$.

Following seven days of enteral nutritional support there was a significant reduction in serum CRP from $156(117-222) \mathrm{mg} / \mathrm{l}$ to 84 $(50-141) \mathrm{mg} / \mathrm{l} \quad(\mathrm{p}<0.005)$ and APACHE II scores fell from $8(6-10)$ to $6(4-8)(\mathrm{p}<0.0001)$ in the enterally fed group (fig 2). However, in the parenterally fed group, no significant change in CRP (125 (49-168) $\mathrm{mg} / \mathrm{l}$ to 124 (73-169) $\mathrm{mg} / \mathrm{l}$ ) or APACHE II scores (9.5 $(8-13)$ to $8(6-12)$ ) occurred (fig 2). The primary assessment CT scores were not significantly different between the two groups (3 (2-5) in both groups) and no significant change was noted in CT scan scores in either the enterally fed $(3(2-5)$ to $3(3-5))$ or the parenterally fed $(3(2-5)$ to $3(2-6))$ groups over the seven days of nutritional support.

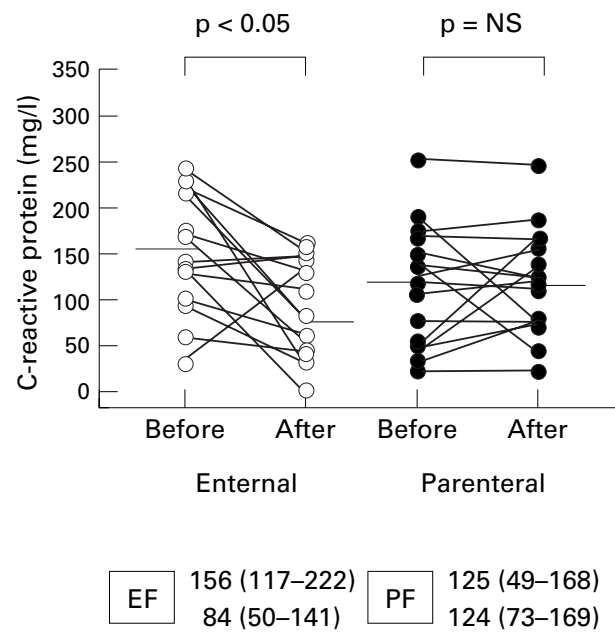


Table 2 Clinical outcome measures

\begin{tabular}{lll}
\hline & Enteral & Parenteral \\
\hline SIRS & 11 pre $v 2$ post $^{\star}$ & 12 pre $v 10$ post \\
Sepsis & 0 & 3 \\
MOF & 0 & 5 \\
& & $(3 \times$ Pulmonary $)$ \\
& & $(2 \times$ Pulmonary, renal and cardiovascular $)$ \\
Operative intervention & $1 \times$ Cystgastrostomy & $1 \times$ Cystgastrostomy \\
& & $2 \times$ Necrosectomy \\
Mortality & 0 & 2 \\
& & $(7$ days and 14 days $)$ \\
\hline
\end{tabular}

${ }^{\star} \mathrm{p}<0.05$ pre- $v$ postnutritional support

SIRS, systemic inflammatory response syndrome; $\mathrm{MOF}$, multisystem organ failure (incidence at end of nutritional support).
Serum IgM EndoCAb antibodies increased by a median of $28.5(24.8-30.7) \%$ following seven days of nutritional support in the parenterally fed group but remained essentially unchanged in those fed enterally $(-1.1(-8.4$ to $+4.41) \%, p<0.05)$. Furthermore, the total antioxidant capacity fell by a median of 27.7 $(-44.9$ to -18.5$) \%$ in the parenterally fed group while increasing by a median of 32.6 $(13.5-45.2) \%$ in the enterally fed patients $(\mathrm{p}<0.05)$.

Clinical outcome measures all improved in the enterally fed patients when compared with the parenterally fed patients. In the enterally fed patients, SIRS was present in 11 prior to nutritional support but in only two patients at the end of nutritional support $(\mathrm{p}<0.05)$ (table 2 ). There was no significant change (12 versus 10) in the incidence of SIRS following seven days of parenteral feeding (table 2). Three patients in the parenterally fed group developed microbiologically proven intra-abdominal sepsis: two with pancreatic infection and one with a liver abscess. The two patients with pancreatic infection required necrosectomy and drainage of the infected material. Five parenterally fed patients also developed organ failure: three with single organ failure (pulmonary) and two with three failed organs (pulmonary, renal, cardiovascular). Two of these patients died, one at seven days without documented sepsis and one at 14 days with evidence of pancreatic necrosis and infection following surgical intervention (table 2). No patient in either group developed catheter related infection on microbiological examination of the intravenous catheter.

Hospital stay was not significantly different between the two groups with patients in the enterally fed group being in hospital for a median of 12.5 (9.5-14) days and in the parenterally fed group for 15 (11-28) days. In contrast, five patients in the parenterally fed group required a median of 10 days on the intensive care unit while no patient in the enterally fed group required intensive care unit management.

\section{Discussion}

Despite the lack of prospective data, conventional wisdom dictates that gut rest with or without the provision of parenteral nutrition remains the treatment of choice in acute pancreatitis. ${ }^{25}$ This study set out to explore the feasibility of enteral feeding in acute pancreatitis and to determine whether it improved clinical outcome and modulated the acute phase response in keeping with other trials of enteral feeding in the critically ill. ${ }^{7-9}$ The first conclusion to be drawn from this study is that enteral feeding in acute pancreatitis is pactical. Enteral nutrition did not have to cease in any patients although a small number of patients with an ileus were unable to tolerate the full volume of feed for brief periods, thus explaining the lower total energy delivery per patient per day in the enterally fed group. The use of antidiarrhoeal agents was avoided in this study by commencing the enteral feeding with a low osmolarity solution and gradually building up to the full strength over a 24 hour period. However, nitrogen delivery was almost identical in the two groups and it might be argued that the clinical benefit derived from the enteral nutrition resulted from direct contact between the intestinal mucosa and nutritional supply even in those patients who developed an ileus. ${ }^{26}$

In the enterally fed group there was an objective reduction in the requirement for ITU care, incidence of intra-abdominal sepsis, multiple organ failure, need for operative intervention, and mortality when compared with the parenterally fed patients. All but one of the parenterally fed patients who required ITU care were admitted from the ward with progression of their disease having already been commenced on nutritional support. The one exception to this was a patient transfered to the ITU within 48 hours of admission with overwhelming disease resulting from a massive inflammatory response leading to rapidly fatal progression to multiple organ failure. It is unlikely that enteral intervention in this case would have altered the eventual clinical outcome.

In addition to the central finding of this study, the data also suggest that enteral feeding provides clinical benefit in terms of acute physiology, acute phase response, morbidity, and mortality. Following seven days of nutritional support, CRP levels fell significantly in the enteral group, taking the postnutritional CRP (84 mg/l) below $120(\mathrm{mg} / \mathrm{l})$, a recognised threshold for severe disease in acute pancreatitis. Similarly, the APACHE II scores fell significantly in the enterally fed group with the postnutritional APACHE II score falling below 8, a further threshold for severe disease according to the Atlanta criteria. These findings seem to corroborate those of Peterson et $a l^{10}$ and Kudsk et $a l^{11}$ in their series of trauma patients, suggesting that enteral feeding modulates the acute phase response while reprioritising hepatic visceral protein metabolism. In contrast, CRP remained unchanged following seven days of parenteral nutrition, with the postnutritional CRP remaining above the threshold for severity of $120 \mathrm{mg} / \mathrm{l}$. Likewise, APACHE II scores in the parenterally fed group showed no significant change and remained above the severity threshold of 8 .

No significant change in CT scores was noted in either group, suggesting that apparent improvements in acute phase response and acute physiology in the enterally fed patients occurred in the absence of measurable changes 
in the extent of pancreatic injury. Thus there was no evidence that any potential effects of the enteral nutrition on pancreatic exocrine function ${ }^{27}$ did not increase the severity of the pancreatitis. The significant $\operatorname{IgM}$ antiendotoxin response in the intravenously fed patients raises the possibility that failure of gut barrier function resulting from inadequate mucosal nutrition may lead to significant systemic exposure to endotoxin. In addition, the reduction in antioxidant capacity noted in the parenterally fed patients is consistent with an increase in endotoxin derived oxidant stress in these patients. In stark contrast to these effects noted in the parenterally fed group, enterally fed patients showed no IgM antibody response and an improved antioxidant capacity following seven days of nutritional support, indicating that feeding the gut may provide some protection against recent systemic endotoxaemia.

The cost implications of these observations as a whole would seem enormous as has been underscored elsewhere. ${ }^{15}$ Although this study failed to identify a difference in hospital stay between the two treatment groups, the need to standardise the investigations for patients with both mild and moderate/severe pancreatitis may have artificially prolonged the hospital stay for the patients with mild disease in order to accommodate the protocol. This may have masked a real advantage for the enterally fed group in terms of hospital stay. However, as a crude estimate parenteral feeding costs an estimated $£ 75$ per day, not including disposables. Enteral feeding costs an estimated $£ 5$, again not including disposables. If one takes into account the need for ITU stay and potential complications in the parenterally fed patients the net cost of parenterally feeding the patients with acute pancreatitis in this study would be considerably more than that for enteral feeding. Even if there had been no significant benefit to clinical or laboratory parameters, the fact that the enterally fed group did not fare less well than their patenterally fed counterparts provides a compelling economic argument in favour of the enteral rather than parenteral route of nutritional support in patients with acute pancreatitis.

An alternative interpretation of these data potentially lies in the nature of the nutritional support administered. It is well recognised that the quantities of vitamins and trace elements which can be added to parenteral nutritional solutions is well below that which can be accommodated in enteral feeds. Moreover, the parenteral group received a higher proportion of long chain triglycerides (LCT) than the enteral group. The immunological effects of LCT remain controversial, ${ }^{28}$ but the combination of fewer antioxidants and greater LCT administration in the parenteral group may theoretically be harmful. Ethical constraints prevented us from including a third arm to this study in which patients recieved only electolyte solutions, which may have gone some way towards answering this question.

In conclusion, this study suggests that contrary to conventional wisdom, enteral feeding is both feasible and desirable in the management of patients with acute pancreatitis. Furthermore, despite the heterogeneity within the patient groups, this study provides evidence that enteral feeding seems to improve disease severity and clinical outcome by modifying the acute phase response and lends support to the concept of identifying the gut as a therapeutic target in the critically ill. The role of enteral nutrition deserves further prospective evaluation, particularly in the subgroup of patients with severe acute pancreatitis.

Part of this work has been published in abstract form (Br F Surg 1997;84:875)

1 Isenman R, Buchler MW. Infection and acute pancreatitis. Br f Surg 1994;81:1707-8.

2 Widdison AL, Karanjia ND. Pancreatic infection complicating acute pancreatitis. Br f Surg 1993;80:148-54.

3 Goris RJ. Multiple organ failure: whole body inflammation? Schweiz Med Wochenschr 1989;119:347-53.

4 Fink MP. Gastrointestinal mucosal injury in experimental models of shock, trauma and sepsis. Crit Care Med 1991;19:627-41

5 Runkel NSF, Moody FG, Smith GS, et al. The role of the gut in the development of sepsis in acute pancreatitis. $\mathcal{F}$ Surg Res 1991;51:18-23.

6 Ryan CM, Schmidt J, Lewandrowski K, et al. Gut macromolecular permeability in pancreatitis correlates with severity of disease in rats. Gastroenterology 1993;104: $890-5$.

7 Moore EE, Jones TN. Benefits of immediate jejunostomy feeding after major abdominal trauma-a prospective randomised trial. F Trauma 1986;26:874-81.

8 Alexander JW, MacMillan BG, Stinnert JD. Beneficial effects of aggressive protein feeding in severely burned children. Surgery 1980;192:505-18.

9 Moore FA, Feliciano DV, Andrassy RJ, et al. Early enteral feeding compared with parenteral reduces postoperative septic complications: the results of a meta-analysis. Ann Surg 1992;216:172-83.

10 Peterson VM, Moore EE, Jones TN, et al. Total enteral nutrition versus total parenteral nutrition after major torso injury: attenuation of hepatic protein reprioritisation. injury: attenuation of hepary $1988 ; 104: 199-207$.

11 Kudsk KA, Minard G, Wojtysiac SL, et al. Visceral protein response to enteral versus parenteral nutrition and sepsis in patients with trauma. Surgery 1994;116:516-23.

12 Kalfarentzos FE, Karavias DD, Karatzas TM, et al. Total parenteral nutrition in severe acute pancreatitis. $\mathcal{F}$ Am Coll Nutr 1991;10:156-62.

3 Forsmark CE, Toskes PP. Acute pancreatitis. Medical management. Crit Care Clin 1995;11:295-309.

14 Marulendra S, Kirby DF. Nutrition support in pancreatitis. Nutrition in Clinical Practice 1995;10:45-53.

15 McClave SA, Greene LM, Snider HL, et al. Comparison of the safety of early enteral vs parenteral nutrition in mild acute pancreatitis. FPEN $\mathcal{F}$ Parenter Enteral Nutr 1997;21: $14-20$.

16 Kudsk KA, Campbell SM, O'Brien T, et al. Postoperative jejunal feeding following complicated pancreatitis. Nutrition in Clinical Practice 1990;5:14-17.

17 Wilson C, Heath DI, Imrie CW. Prediction of outcome in acute pancreatitis: a comparative study of APACHE II, clinical assessment and multiple factor scoring systems. $\mathrm{Br}$ 7 Surg 1990;77:1260-4.

8 Knaus WA, Draper EA, Wagner DP, et al. APACHE II: a severity of disease classification system. Crit Care Med 1985;13:818-29.

19 Barclay GR. Antibodies to endotoxin in health and disease. Rev Med Microbiol 1990;1:133-42.

20 Whitehead TP, Thorpe GHG, Maxwell SRJ. Enhancement chemiluminescent assay for antioxidant capacity. Analyses Chimica Acta 1992;266:265-77.

21 Balthazar EJ. CT diagnosis and staging of acute pancreatitis. Radiol Clin North Am 1989;27:19-37.

22 Balthazar EJ, Robinson DL, Bow AJM, et al. Acute Balthazar EJ, Robinson DL, Bow AJM, et al. Acute pancreatitis: value of CT

23 Bone RC, Sibbald WJ, Sprung CL. The ACCP-SCCM consensus conference on sepsis and organ failure. Chest 1992;101:1481-3.

24 Tran DD, Groeneveld AB, Meulen JVD, et al. Age, chronic disease, sepsis, organ system failure and mortality in a medical intensive care unit. Crit Care Med 1990;18:474-9.

25 Pisters PWT, Ranson JHC. Nutritional support for acute pancreatitis. Surg Gynecol Obstet 1992;175:275-84.

6 Anderson ID, Fearon KCH. Paralytic ileus and enteral feeding. British fournal of Intensive Care 1995;3:117-21.

27 Bodoky G, Harsanyi L, Pap A, et al. Effect of enteral nutrition on exocrine function. Am f Surg 1991;161:144-8.

28 Guillou PJ, Sedman PC, Monson JRT, et al. Modifications of lymphocyte function by fatty acids. In: Cunninghamof lymphocyte function by fatty acids. In: Cunningham-
Rundles S, ed. Nutritional modulation of the immune response. New York: Marcel Dekker, 1992:369-91. 